\title{
The Odd-Point Ternary Approximating Schemes
}

\author{
Ghulam Mustafa*, Abdul Ghaffar, Faheem Khan \\ Department of Mathematics, The Islamia University of Bahawalpur Pakistan, Bahawalpur, Pakistan \\ E-mail: \{mustafa rakib,gulzarkhan143\}@yahoo.com,fahimscholar@gmail.com \\ Received April 1, 2011; revised May 3, 2011; accepted May 15, 2011
}

\begin{abstract}
We present a general formula to generate the family of odd-point ternary approximating subdivision schemes with a shape parameter for describing curves. The influence of parameter to the limit curves and the sufficient conditions of the continuities from $C^{0}$ to $C^{5}$ of 3- and 5-point schemes are discussed. Our family of 3-point and 5-point ternary schemes has higher order of derivative continuity than the family of 3-point and 5-point schemes presented by [16]. Moreover, a 3-point ternary cubic B-spline is special case of our family of 3-point ternary scheme. The visual quality of schemes with examples is also demonstrated.
\end{abstract}

Keywords: Approximating Subdivision Scheme, Derivative Continuity, Smoothness Convergence, Shape Parameters, Laurent Polynomial

\section{Introduction}

Subdivision schemes are important and powerful tools for generation of smooth curves and surfaces from a set of control points by means of iterative refinement. Their popularity is due to the facts that subdivision algorithms are easy to implement and suitable for computer applications. If the limit curve/surface approximate the initial control polygon and that after subdivision, the newly generated control points are not in the limit curve/ surface, the scheme is said to be approximating. It is called interpolating if after subdivision, the control points of the original control polygon and the new generated control points are interpolated on the limit curve/surface.

Beccari et al. [1] introduced an interpolating 4-point $C^{2}$ ternary non-stationary subdivision scheme with tension control. Hassan and Dodgson [2] presented ternary and three-point univariate subdivision schemes. Khan and Mustafa [3] offered ternary six-point interpolating subdivision scheme. Ko et al. [4] presented a ternary 4-point approximating subdivision scheme. Dyn [5] gave the analysis of interpolatory subdivision schemes by the formalism of Laurent polynomials. [6,7] and [8] also introduced the analysis of the scheme by Laurent polynomials methods. Sabin [9] has presented eigenanalysis and artifacts of subdivision curves and surfaces. Levin [10] has presented the polynomial generation and quasi interpolation in stationary non-uniform subdivision schemes. Hormann et al. [11] introduced a family of subdivision schemes with cubic precision. Dyn et al. [12] have presented polynomial reproduction by symmetric schemes.

Since higher arity schemes have very nice properties (i.e. high smoothness, high approximation order and lower support) than their counterpart of lower arity schemes. Therefore research communities are gaining interest in introducing higher arity schemes (i.e. ternary, quaternary, ..., $a$-ary). Mustafa and Khan [13] offered a new 4point $C^{3}$ quaternary approximating subdivision scheme. Lian [14] generalized classical 4-point and 6-point interpolating schemes to $a$-ary interpolating schemes for any integer $a \geq 3$. These new $a$-ary schemes are derived from corresponding two scale functions, a notion from the content of wavelets. Lian [15] has also introduced $a$-ary 3-point and 5-point interpolating schemes for arbitrary odd integer $a \geq 3$. Unfortunately, schemes presented by [15] have very stumpy smoothness that is Lian's 3- and 5-point schemes have $C^{1}$ continuity while schemes introduced in this article have $C^{2}$ and $C^{5}$ continuity respectively. Lian [16] also offered $2 m$-point and $(2 m+1)$-point interpolating $a$-ary schemes for curve design. Mustafa and Rehman [17] introduced the explicit formulae to generate the mask of $(2 b+4)$-point $n$-ary subdivision scheme. Siddiqi and Rehan [18] introduced modified form of binary and ternary 3-point subdivision schemes which are $C^{1}$ and $C^{2}$ in the intervals $\left(\frac{-1}{8}, \frac{5}{24}\right)$ and $\left(\frac{-1}{72}, \frac{7}{72}\right)$ respectively. These 
intervals are too narrow to provide freedom for curve designing. This motivates us to present the family of odd-point ternary schemes with high smoothness and more degree of freedom for curve designing.

The paper is organized as follows: we recall basic definitions and preliminary results in Section 2. The family of odd-point ternary approximating schemes and analysis by Laurent formalism of one odd-point ternary scheme is presented in Section 3. Basic properties of odd-point ternary schemes are discussed in Section 4. Comparison with existing odd-point ternary schemes is also shown in this section. A few remarks and future work constitute Section 5.

\section{Preliminaries}

A general compact from of univariate ternary subdivision scheme $S$ which maps polygon $f^{k}=\left\{f_{i}^{k}\right\}_{i \in Z}$ to a refined polygon $f^{k+1}=\left\{f_{i}^{k+1}\right\}_{i \in Z}$ is defined by

$$
f_{i}^{k+1}=\sum_{j \in Z} \alpha_{3 j-i} f_{i}^{k}, \quad i \in Z,
$$

where the set $a=\left\{a_{i}: i \in Z\right\}$ of coefficients is called the mask at $k t h$ level of refinement. A necessary condition for the uniform convergence of subdivision scheme (2.1) is that

$$
\sum_{j \in Z} \alpha_{3 j}=\sum_{j \in Z} \alpha_{3 j+1}=\sum_{j \in Z} \alpha_{3 j+2}=1 .
$$

A subdivision scheme is uniformly convergent if for any initial data $f^{0}=\left\{f_{i}^{o}: i \in Z\right\}$, there exists a continuous function $f$ such that for any closed interval $I \subset R$, satisfies

$$
\lim _{k \rightarrow \infty} \sup _{i \in 3^{k} I}\left|f_{i}^{k}-f\left(3^{-k} i\right)\right|=0 .
$$

Obviously, $f=S^{\infty} f^{0}$.

For analysis of scheme, the z-transform of the mask

$$
\alpha(z)=\sum_{i \in Z} a_{i} z^{i},
$$

which is usually called the Laurent polynomial of scheme and plays a crucial role in the analysis of the scheme. From (2.2) and (2.3) the Laurent polynomial of convergent subdivision scheme satisfies

$$
a\left(e^{2 i \pi / 3}\right)=a\left(e^{4 i \pi / 3}\right)=0 \text { and } a(1)=3 .
$$

This condition guarantees the existence of a related subdivision scheme for the divided differences of the original control points and the existence of an associated Laurent polynomial $a^{(1)}(z)$

$$
a^{(1)}(z)=\frac{3 z^{2}}{1+z+z^{2}} a(z) .
$$

The subdivision scheme $S_{1}$ with Laurent polynomial $a^{(1)}(z)$, is related to scheme $S$ with Laurent polynomial $a(z)$ by the following theorem.

Theorem 2.1. [1] Let S denote a subdivision scheme with Laurent polynomial $a(z)$ satisfying (2.4). Then there exists a subdivision scheme $S_{1}$ with the property

$$
\Delta f^{k}=S_{1} \Delta f^{k-1}, a(z)
$$

where $f^{k}=S^{k} f^{0}$ and $\Delta f^{k}=\left\{\left(\Delta f^{k}\right)_{i}=3^{k}\left(f_{i+1}^{k}-f_{i}^{k}\right)\right.$; $i \in Z$ \} . Furthermore, $S$ is a uniformly convergent if and only if $\frac{1}{3} S_{1}$ converges uniformly to zero function for all initial data $f^{0}$, in the sense that

$$
\lim _{k \rightarrow 0}\left(\frac{1}{3} S_{1}\right)^{k} f^{0}=0
$$

The above theorem indicates that for any given scheme $S$, with mask ' $a$ '. satisfying (2.2), we can prove the uniform convergence of $S$ by deriving the mask of $\frac{1}{3} S_{1}$ and computing $\left\|\left(\frac{1}{3} S_{1}\right)^{i}\right\|_{\infty}$ for $i=1,2,3, \cdots, L$, where $L$ is the first integer for which $\left\|\left(\frac{1}{3} S_{1}\right)^{L}\right\|<1$. If such an $L$ exists, then $S$ converges uniformly. Since there are three rules for computing the values at next refinement level, so we define the norm

$$
\|S\|_{\infty}=\max \left\{\sum_{j \in Z}\left|a_{3 j}\right|, \sum_{j \in Z}\left|\alpha_{3 j+1}\right|, \sum_{j \in Z}\left|a_{3 j+2}\right|\right\},
$$

and

$$
\left\|\left(\frac{1}{3} S_{n}\right)^{L}\right\|=\max \left\{\sum_{j \in Z}\left|b_{i+3^{L} j}^{[n, L]}\right| ; i=0,1,2, \cdots, 3^{L}-1\right\},
$$

where

$$
b^{[n, L]}(z)=\frac{1}{3^{L}} \prod_{j=1}^{L-1} a^{(n)}\left(z^{3 j}\right),
$$

and

$$
\begin{gathered}
a^{(n)}(z)=\left(\frac{3 z^{2}}{1+z+z^{2}}\right) a^{(n-1)}(z)=\left(\frac{3 z^{2}}{1+z+z^{2}}\right)^{n} a(z), \\
n \geq 1 .
\end{gathered}
$$

Theorem 2.2. [6] Let $S$ be subdivision scheme with a characteristic polynomial $a(z)=\left(\frac{1+z+z^{2}}{3 z^{2}}\right)^{n} q(z)$. If the subdivision scheme $S_{n}$ corresponding to the polynomial $q(z)$ converges uniformly, then $S^{\infty} f^{0} \in$ $C^{n}(R)$ for any initial control polygon $f^{0}$. 
Corollary 2.3. [6] If $S$ is a subdivision scheme of the form above and $\frac{1}{3} S_{n+1}$ converges uniformly to the zero function for all initial data $f^{0}$ then $S^{\infty} f^{0} \in C^{n}(R)$ for any initial control polygon $f^{0}$.

Corollary 2.3 indicates that for any given ternary subdivision scheme $S$, we can prove $S^{\infty} f^{0} \in C^{n}(R)$ by first deriving the mask of $\frac{1}{3} S_{n+1}$ and then computing $\left\|\left(\frac{1}{3} S_{n+1}\right)^{i}\right\|_{\infty}$ for $i=1,2,3, \cdots, L$, where $L$ is the first integer for which $\left\|\left(\frac{1}{3} S_{n+1}\right)^{L}\right\|_{\infty}<1$ If such an $L$ exists, then $S^{\infty} f^{0} \in C^{n}(R)$.

\section{The Odd-Point Ternary Approximating Schemes}

Here we propose the general formula for odd-point ternary approximating subdivision schemes with one parameter in the form of Laurent polynomial

$$
\begin{aligned}
a_{[\mu]}(z)= & \frac{1}{3^{\mu+n-1}}\left(1+z+z^{2}\right)^{\mu+n} \\
& \left\{\left(\frac{1}{12}+\omega\right)+\left(\frac{5}{6}-2 \omega\right) z+\left(\frac{1}{12}+\omega\right) z^{2}\right\}
\end{aligned}
$$

where $\mu=2 n+3, n \geq 0$. Although one can easily generate $(2 n+3)$-point ternary schemes for $n \geq 0$ from (3.1), for simplicity, we generate and discuss the smoothness of only 3-point ternary scheme. The smoothness of other odd-point ternary schemes can be computed in similar way. Moreover, for $n=0$, $\omega=1 / 4$ (3.1) simplifies to $[1,4,10,16,19,16,10$, $4,1]$ / 27 which is just 3-point ternary cubic B-spline.

\subsection{A 3-Point Ternary Scheme}

From (3.1) for $n=0$, we get Laurent polynomial for 3-point scheme

$$
\begin{aligned}
a_{[3]}(z)= & \frac{1}{9}\left\{\left(\frac{1}{12}+\omega\right)+\left(\frac{13}{12}+\omega\right) z+\left(\frac{37}{12}+\omega\right) z^{2}\right. \\
& +\left(\frac{35}{6}-2 \omega\right) z^{3}+\left(\frac{41}{6}-2 \omega\right) z^{4}+\left(\frac{35}{6}-2 \omega\right) z^{5} . \\
& \left.+\left(\frac{37}{12}+\omega\right) z^{6}+\left(\frac{13}{12}+\omega\right) z^{7}+\left(\frac{1}{12}+\omega\right) z^{8}\right\}
\end{aligned}
$$

This gives the mask of 3-point scheme

$$
\begin{aligned}
a_{[3]}= & \frac{1}{9}\left[0, \cdots, 0,0,\left(\frac{1}{12}+\omega\right),\left(\frac{13}{12}+\omega\right),\left(\frac{37}{12}+\omega\right),\right. \\
& \left(\frac{35}{6}-2 \omega\right),\left(\frac{41}{6}-2 \omega\right),\left(\frac{35}{6}-2 \omega\right), \\
& \left.\left(\frac{37}{12}+\omega\right),\left(\frac{13}{12}+\omega\right),\left(\frac{1}{12}+\omega\right), 0,0, \cdots, 0\right]
\end{aligned}
$$

From the above mask, we suggest following 3-point ternary approximating scheme

$$
\begin{aligned}
& f_{3 i}^{k+i}=\frac{1}{9}\left[\begin{array}{l}
\left(\frac{37}{12}+\omega\right) f_{i-1}^{k}+\left(\frac{35}{6}-2 \omega\right) f_{i}^{k} \\
+\left(\frac{1}{12}+\omega\right) f_{i+1}^{k}
\end{array}\right], \\
& f_{3 i+1}^{k+i}=\frac{1}{9}\left[\begin{array}{l}
\left(\frac{13}{12}+\omega\right) f_{i-1}^{k}+\left(\frac{41}{6}-2 \omega\right) f_{i}^{k} \\
+\left(\frac{13}{12}+\omega\right) f_{i+1}^{k}
\end{array}\right], \\
& f_{3 i+2}^{k+i}=\frac{1}{9}\left[\begin{array}{l}
\left(\frac{1}{12}+\omega\right) f_{i-1}^{k}+\left(\frac{35}{6}-2 \omega\right) f_{i}^{k} \\
+\left(\frac{37}{12}+\omega\right) f_{i+1}^{k}
\end{array}\right] .
\end{aligned}
$$

From (2.8), we have

$$
a_{[3]}^{(1)}(z)=\left(\frac{3 z^{2}}{1+z+z^{2}}\right) a_{[3]}(z) .
$$

This implies

$$
a_{[3]}^{(1)}=\frac{z^{2}}{3}\left\{\begin{array}{l}
\left(\frac{1}{12}+\omega\right)+z+2 z^{2}+ \\
\left(\frac{17}{6}-2 \omega\right) z^{3}+2 z^{4}+z^{5}+\left(\frac{1}{12}+\omega\right) z^{6}
\end{array}\right\}
$$

and

$$
a_{[3]}^{(1)}=\frac{1}{3}\left\{\begin{array}{l}
0, \cdots, 0,0,\left(\frac{1}{12}+\omega\right), 1,2,\left(\frac{17}{6}-2 \omega\right), \\
2,1,\left(\frac{1}{12}+\omega\right), 0,0, \cdots, 0
\end{array}\right\}
$$

From (2.7), we have

$$
b^{[1,1]}=\frac{1}{3} a_{[3]}^{(1)}(z)=\frac{1}{3}\left(\frac{3 z^{2}}{1+z+z^{2}}\right) a_{[3]}(z) .
$$

This implies

$$
b^{[1,1]}=\frac{z^{2}}{9}\left\{\begin{array}{l}
\left(\frac{1}{12}+\omega\right)+z+2 z^{2}+\left(\frac{17}{6}-2 \omega\right) z^{3} \\
+2 z^{4}+z^{5}+\left(\frac{1}{12}+\omega\right) z^{6}
\end{array}\right\} .
$$


This can be written as

$$
b^{[1,1]}=\frac{1}{9 z^{5}}\left\{\begin{array}{l}
\left(\frac{1}{12}+\omega\right) z^{-3}+z^{-2} \\
+2 z^{-1}+\left(\frac{17}{6}-2 \omega\right) z^{0} \\
+2 z^{1}+z^{2}+\left(\frac{1}{12}+\omega\right) z^{3}
\end{array}\right\}
$$

If $S_{1}^{[3]}$ is the scheme corresponding to $a_{[3]}^{(1)}$, then for $C^{0}$ continuity, we require that $a_{[3]}^{(1)}$ satisfies (2.2), which it does and $\left\|\left(\frac{1}{3} S_{1}^{[3]}\right)^{L}\right\|_{\infty}<1$ Since from (2.6), for $L=1$, we have

$$
\left\|\left(\frac{1}{3} S_{1}\right)\right\|=\max \left\{\sum_{j \in Z}\left|b_{i+3 j}^{[1,1]}\right| ; i=0,1,2\right\} .
$$

This implies that

$$
\left\|\left(\frac{1}{3} S_{1}\right)\right\|=\max \left\{\sum_{j \in Z}\left|b_{i+3 j}^{[1,1]}\right| ; i=0,1,2\right\} .
$$

for integer values of $j \quad(-3 \leq 3 j \leq 3)$ that is $j=-1,0,1$ and from (3.3), we get

$$
\begin{aligned}
\sum_{j=-1}^{1}\left|b_{3 j}^{[1,1]}\right| & =\left|b_{-3}\right|+\left|b_{0}\right|+\left|b_{3}\right| \\
& =\frac{1}{9}\left|\frac{1}{12}+\omega\right|+\frac{1}{9}\left|\frac{17}{6}-2 \omega\right|+\left|\frac{1}{12}+\omega\right|, \\
& =\frac{2}{9}\left|\frac{1}{12}+\omega\right|+\frac{1}{9}\left|\frac{17}{6}-2 \omega\right| \\
\sum_{j=-1}^{1}\left|b_{1+3 j}^{[1,1]}\right| & =\left|b_{-2}\right|+\left|b_{1}\right|+\left|b_{4}\right|=\left|\frac{1}{9}\right|+\left|\frac{2}{9}\right|+0=\frac{1}{3},
\end{aligned}
$$

and

$$
\sum_{j=-1}^{1}\left|b_{2+3 j}^{[1,1]}\right|=\left|b_{-1}\right|+\left|b_{2}\right|+\left|b_{5}\right|=\left|\frac{2}{9}\right|+\left|\frac{1}{9}\right|+0=\frac{1}{3} .
$$

Summarizing, we get following for $-\frac{19}{12}<\omega<\frac{35}{12}$

$$
\begin{gathered}
\left\|\left(\frac{1}{3} S_{1}^{[3]}\right)\right\|_{\infty}= \\
\max \left\{\frac{2}{9}\left|\frac{1}{12}+\omega\right|+\frac{1}{9}\left|\frac{17}{6}-2 \omega\right|, \frac{1}{3}\right\}<1
\end{gathered}
$$

Then by Theorem 2.1, 3-point scheme is $C^{0}$. From (2.8), we have

$$
a_{[3]}^{(2)}(z)=\left(\frac{3 z^{2}}{1+z+z^{2}}\right) a_{[3]}^{(1)}(z) .
$$

This implies

$$
a_{[3]}^{(2)}(z)=z^{4}\left\{\begin{array}{l}
\left(\frac{1}{12}+\omega\right)+\left(\frac{11}{12}-2 \omega\right) z+z^{2} \\
+\left(\frac{11}{12}-2 \omega\right) z^{3}+\left(\frac{1}{12}+\omega\right) z^{4}
\end{array}\right\},
$$

and

$$
a_{[3]}^{(2)}=\frac{1}{3}\left\{\begin{array}{l}
0, \cdots, 0,0,\left(\frac{1}{12}+\omega\right),\left(\frac{11}{12}-2 \omega\right), \\
1,\left(\frac{11}{12}-2 \omega\right),\left(\frac{1}{12}+\omega\right), 0,0, \cdots, 0
\end{array}\right\} .
$$

If $S_{2}^{[3]}$ is the scheme corresponding to $a_{[3]}^{(2)}$, then for $C^{1}$ continuity, we require that $a_{[3]}^{(2)}$ satisfies (2.2), which it does and $\left\|\left(\frac{1}{3} S_{2}^{[3]}\right)^{L}\right\|_{\infty}<1$. Since from (2.6), for $\frac{-13}{18}<\omega<\frac{23}{18}$ and $L=1$, we have

$$
\begin{gathered}
\left\|\left(\frac{1}{3} S_{2}^{[3]}\right)\right\|_{\infty}= \\
\max \left\{\frac{1}{3}\left|\frac{1}{12}+\omega\right|+\frac{1}{3}\left|\frac{11}{12}-2 \omega\right|, \frac{1}{3}\right\}<1
\end{gathered}
$$

then by Corollary 2.3, 3-point scheme is $C^{1}$. From (2.8) we have

$$
a_{[3]}^{(3)}(z)=\left(\frac{3 z^{2}}{1+z+z^{2}}\right) a_{[3]}^{(2)}(z) .
$$

This implies

$$
a_{[3]}^{(3)}(z)=z^{4}\left\{\begin{array}{l}
\left(\frac{1}{12}+\omega\right)+\left(\frac{11}{12}-2 \omega\right) z+z^{2} \\
+\left(\frac{11}{12}-2 \omega\right) z^{3}+\left(\frac{1}{12}+\omega\right) z^{4}
\end{array}\right\},
$$

and

$$
a_{[3]}^{(3)}=\frac{1}{3}\left\{\begin{array}{l}
0, \cdots, 0,0,\left(\frac{1}{12}+\omega\right), \\
\left(\frac{5}{6}-2 \omega\right),\left(\frac{1}{12}+\omega\right), 0,0, \cdots, 0
\end{array}\right\} .
$$

If $S_{3}^{[3]}$ is the scheme corresponding to $a_{[3]}^{(3)}$, then for $C^{2}$ continuity, we require that $a_{[3]}^{(3)}$ satisfies (2.2), which it does and $\left\|\left(\frac{1}{3} S_{3}^{[3]}\right)^{L}\right\|_{\infty}<1$. Since from (2.6), for $\frac{-1}{12}<\omega<\frac{11}{12}$ and $L=1$, we have 


$$
\left\|\left(\frac{1}{3} S_{3}^{[3]}\right)^{L}\right\|_{\infty}=\max \left\{\left|\frac{1}{12}+\omega\right|,\left|\frac{5}{6}-2 \omega\right|\right\}<1,
$$

then by Corollary 2.3, 3-point scheme is $C^{2}$.

Remark: The continuity of 5-point ternary scheme can be computed in a similar fashion. The sufficient conditions for the order of continuity of proposed 3-point and 5-point ternary schemes for certain ranges of parameter are given in Table $\mathbf{1}$.

\section{Basic Properties of the Schemes}

In this section, we discuss basic properties of odd-point ternary approximating subdivision schemes that are their precision set and support of basic limits function.

\subsection{Precision Set}

Here, we find the precision set of 3-point ternary scheme.

Lemma 4.1. The proposed 3-point ternary precision set scheme has quadratic precision for $\forall \omega$ and cubic at $\omega=\frac{1}{4}$.

Proof. We carry out this result by taking our origin the middle of an original span with ordinate $\cdots,(-3)^{n}$, $(-1)^{n},(1)^{n},(3)^{n}, \cdots$

If $y=x^{n}$, then we have

$$
\begin{aligned}
{[y]=} & \cdots, a_{1}(-3)^{n}+a_{2}(-1)^{n}+a_{3}(1)^{n}, a_{4}(-3)^{n} \\
& +a_{5}(-1)^{n}+a_{4}(1)^{n}, a_{3}(-3)^{n}+a_{2}(-1)^{n} \\
& +a_{1}(1)^{n}, a_{1}(-1)^{n}+a_{2}(1)^{n}+a_{3}(3)^{n}, a_{4}(-1)^{n} \\
& +a_{5}(1)^{n}+a_{4}(3)^{n}, a_{3}(-1)^{n}+a_{2}(1)^{n}+a_{1}(3)^{n}, \cdots
\end{aligned}
$$

where $\quad a_{1}=\frac{1}{9}\left(\frac{37}{12}+\omega\right), \quad a_{2}=\frac{1}{9}\left(\frac{35}{6}-2 \omega\right), \quad a_{3}=\frac{1}{9}$ $\left(\frac{1}{12}+\omega\right), a_{4}=\frac{1}{9}\left(\frac{13}{12}+\omega\right), a_{5}=\frac{1}{9}\left(\frac{41}{6}-2 \omega\right)$,

If $y=x^{1}$, then

$$
\begin{gathered}
{[y]=\cdots-\frac{5}{3},-1,-\frac{1}{3}, \frac{1}{3}, 1, \frac{5}{3}, \cdots} \\
\delta y=\cdots, \frac{2}{3}, \frac{2}{3}, \frac{2}{3}, \frac{2}{3}, \frac{2}{3}, \cdots,
\end{gathered}
$$

\begin{tabular}{|c|c|c|c|c|c|}
\hline Scheme & Parameter & Continuity & yScheme & Parameter & Continuity \\
\hline \multirow[t]{2}{*}{ 3-point - } & $-\frac{19}{12}<\omega<\frac{35}{12}$ & $C^{0}$ & 5-point & $-\frac{28}{3}<\omega<\frac{131}{12}$ & $C^{0}$ \\
\hline & $-\frac{13}{18}<\omega<\frac{23}{18}$ & $C^{1}$ & & $-\frac{71}{12}<\omega<\frac{91}{12}$ & $C^{1}$ \\
\hline \multirow[t]{4}{*}{........ } & $-\frac{1}{12}<\omega<\frac{11}{12}$ & $C^{2}$ & & $-\frac{67}{12}<\omega<\frac{89}{12}$ & $C^{2}$ \\
\hline & & & & $-\frac{19}{12}<\omega<\frac{35}{12}$ & $C^{3}$ \\
\hline & & & .. & $-\frac{13}{18}<\omega<\frac{23}{18}$ & $C^{4}$ \\
\hline & & & .......... & $-\frac{1}{12}<\omega<\frac{11}{12}$ & $C^{5}$ \\
\hline
\end{tabular}

$\left[\delta^{2} y\right]=0$, where $\delta$ represents the differences of the vertices.

$$
\begin{aligned}
& \text { If } \begin{array}{r}
y=x^{2} \text {, then } \\
{[y]=\cdots, \frac{101}{27}+\frac{8}{9} \omega, \frac{29}{27}+\frac{8}{9} \omega, \frac{29}{27}+\frac{8}{9} \omega,} \\
\frac{29}{27}+\frac{8}{9} \omega, \frac{29}{27}+\frac{8}{9} \omega, \frac{101}{27}+\frac{8}{9} \omega, \cdots
\end{array}
\end{aligned}
$$

Table 1. The order of continuities of proposed 3-point and 5 -point ternary approximating schemes are given below.

Taking further differences, we get $\left[\delta^{3} y\right]=0$. If $y=x^{3}$, then

$$
\begin{gathered}
{[y]=\cdots, \frac{-89}{9}-\frac{8}{3} \omega, \frac{-35}{27}-\frac{8}{3} \omega, \frac{-5}{9}-\frac{8}{3} \omega,} \\
\frac{5}{9}+\frac{8}{3} \omega, \frac{35}{9}+\frac{8}{3} \omega, \frac{89}{9}+\frac{8}{3} \omega, \cdots
\end{gathered},
$$

by taking further differences, we have

$$
\begin{gathered}
{\left[\delta^{4} y\right]=\cdots, 4-16 \omega,-4+16 \omega, \cdots,\left[\delta^{4} y\right]=0,} \\
\text { at } \omega=\frac{1}{4},
\end{gathered}
$$

Thus by [9], the proposed scheme has quadratic precision $\forall \omega$ and cubic at $\omega=\frac{1}{4}$. Similarly one can easily prove that proposed 5-point ternary approximating subdivision scheme has quintic (i.e. 5) precision set for $\forall \omega$ and sextic (i.e. 6$)$ at $\omega=\frac{1}{4}$.

\subsection{Remark}

Actually, due to the referee's implication/allusion, we can find the approximation order of proposed 3-point ternary scheme by taking $\omega=\frac{1}{4}$. The mask of 3-point scheme at $\omega=\frac{1}{4}$ simplifies to $[1,4,10,16,19,16$, $10,4,1] / 27$, which is just the ternary cubic B-spline. Now according to the precision analysis this scheme has cubic precision, which is totally correct because cubic polynomials are special case of cubic B-spline, of course. However, B-splines are well-known to have approximation order $o\left(h^{2}\right)$. Here it is pointed that the presented version of the paper owes much to the precise and kind remarks of the anonymous referee.

\subsection{Support of Basic Limit Function}

The basic function of a subdivision scheme is the limit 
function of proposed scheme for the following data

$$
f_{i}^{0}= \begin{cases}1, & i=0, \\ 0, & i \neq 0 .\end{cases}
$$

Figure 1 (a) and (b) show the basic limit functions, $\phi^{[\mu]}=\left(S^{[\mu]}\right)^{\infty} f_{i}^{0}$ of proposed $\mu$-point ternary approximating schemes, for $\mu=2 n+3, n=0,1$ respectively. The following theorem is related to the support of basic limit functions for odd-point ternary schemes.

Theorem 4.4. The basic limit functions $\phi^{[\mu]}$ of pro-

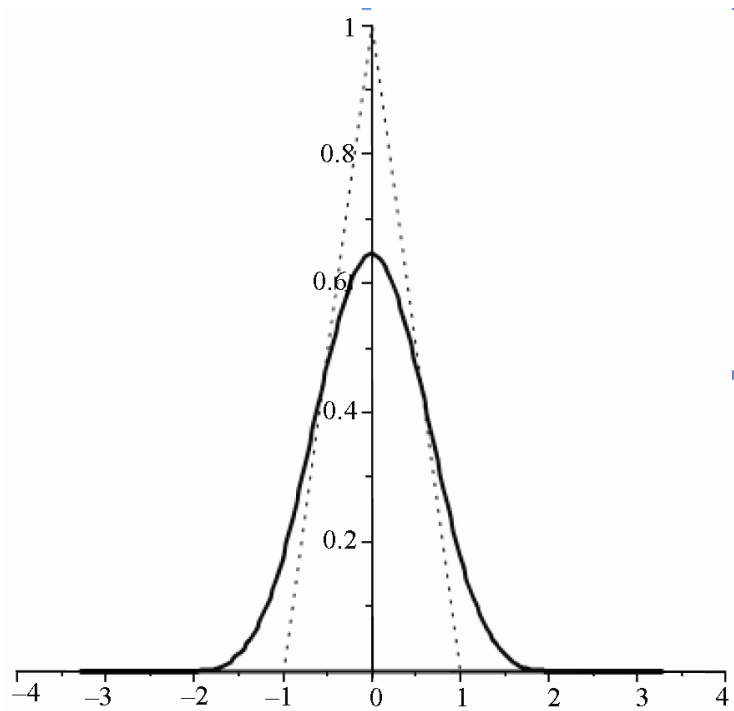

(a)

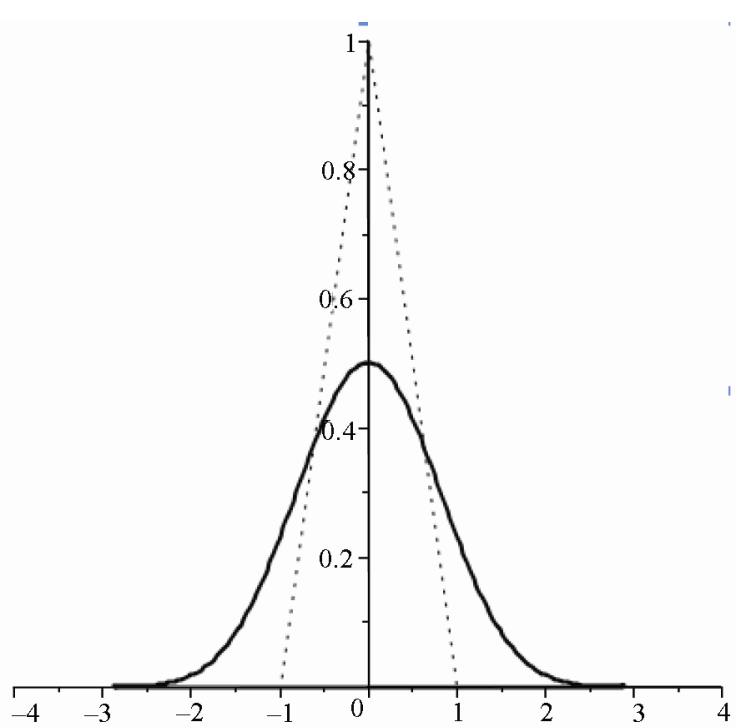

(b)

Figure 1. The basic limit functions of proposed schemes at $\omega=\frac{1}{12}$. (a) 3-point scheme; (b) 5-point scheme. posed $\mu$-point ternary approximating scheme has support width $s=\mu+n+1$, for $\mu=2 n+3, n \geq 0$, which implies that it vanishes outside the interval $\left[-\frac{\mu+n+1}{2}\right.$, $\left.\frac{\mu+n+1}{2}\right]$

Proof. Since the basic function is the limit function of the scheme for the data (4.1), its support width ' $s$ ' can be determine by computing how for the effect of the non-zero vertex $f_{0}^{0}$ will propagate along by. As the mask of $\mu$-point scheme is $3 \mu$-long sequence by centering it on that vertex, the distances to the last of its left and right non-zero coefficients are equal to $\mu+1$.

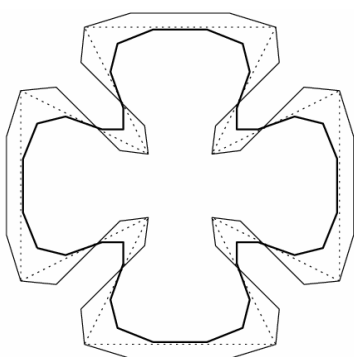

(a)

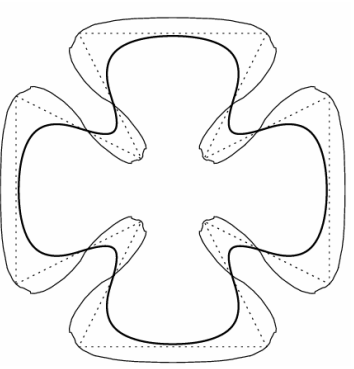

(c)

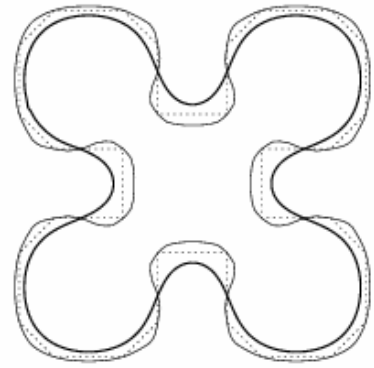

(e)

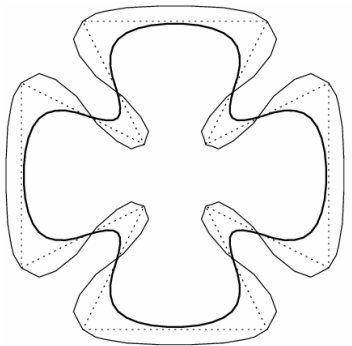

(b)

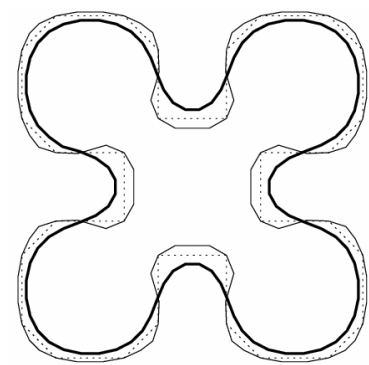

(d)

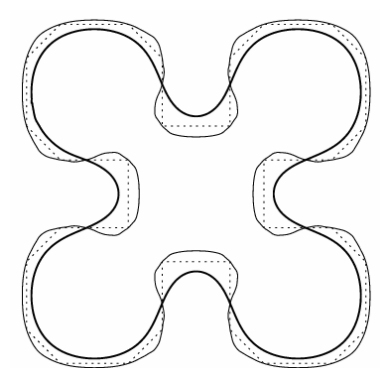

(f)

Figure 2. Comparison: Dotted lines indicate initial polygons. Thin solid and bold solid continuous curves are generated by proposed ternary approximating scheme and Lian [15] ternary interpolating schemes respectively. (a), (b), and (c) show different levels of 3-point ternary schemes, whereas (d), (e), and (f) show different levels of 5-point ternary schemes. 
Table 2. Comparison of proposed 3- and 5-point ternary schemes.

\begin{tabular}{|c|c|c|c|c|c|}
\hline Scheme & Type & Support & Order & $C^{n}$ & Range \\
\hline 3-point ternary [2] & Interpolating & 4 & 2 & $C^{1}$ & $a=b-\frac{1}{3} \quad$ and $\quad b \in\left[\frac{2}{9}, \frac{3}{9}\right]$ \\
\hline 3-point ternary [15] & Interpolating & 4 & 2 & $C^{1}$ & For some particular value \\
\hline 3-point ternary [2] & Approximating & 4 & 2 & $C^{2}$ & For some particular value \\
\hline 3-point ternary [18] & Approximating & 4 & 2 & $C^{2}$ & $\mu \in\left[\frac{-1}{72}, \frac{7}{72}\right]$ \\
\hline 4-point ternary [6] & Interpolating & 5 & 4 & $C^{2}$ & $\mu \in\left[\frac{1}{9}, \frac{1}{15}\right]$ \\
\hline 4-point ternary [4] & Approximating & 5.5 & 4 & $C^{2}$ & For some particular value \\
\hline 5-point ternary [15] & Interpolating & 7 & 4 & $C^{1}$ & For some particular value \\
\hline 3-point ternary proposed & Approximating & 4 & 2 & $C^{2}$ & $-\frac{1}{12}<\omega<\frac{11}{12}$ \\
\hline 5-point ternary proposed & Approximating & 7 & 4 & $C^{5}$ & $-\frac{1}{12}<\omega<\frac{11}{12}$ \\
\hline
\end{tabular}

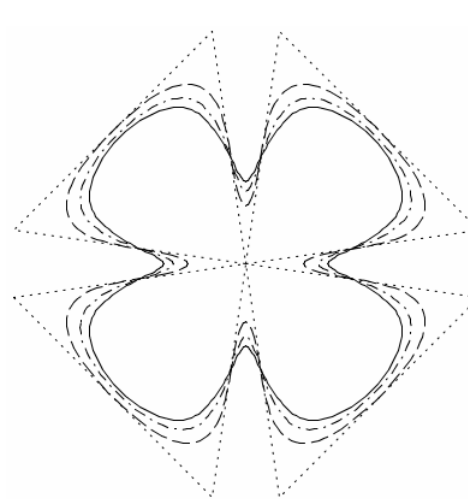

(a)

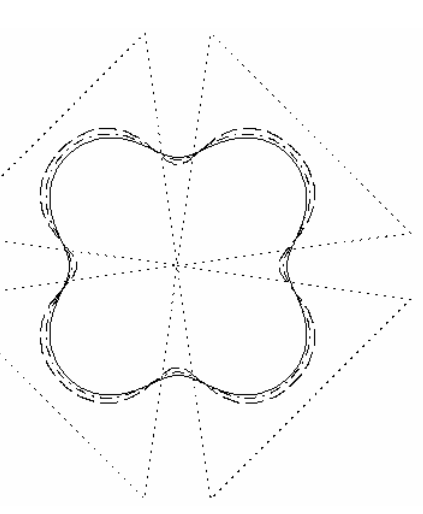

(b)

Figure 3. Dotted lines indicate initial polygons. Dashes, dashes dot and solid line show the visual smoothness of proposed schemes for the parametric value at $\omega=\frac{-1}{12}, \omega=\frac{1}{6}$ and $\omega=\frac{1}{3}$ respectively. (a) 3-point scheme; (b) 5-point scheme.

At the first subdivision step, we see that the vertices on the both sides of $f_{0}^{1}$ at $\frac{\mu+n+1}{3}$ are the furthest nonzero new vertices. At each refinement, the distances on both sides are reduced by the factor $\frac{1}{3}$. At the next step of the scheme this will propagate along by $\frac{\mu+n+1}{3} \cdot \frac{1}{3}$ on both sides. Hence after $k$ subdivision steps the furthest non-zero vertex on the left will be at $(\mu+n+1)\left(\frac{1}{3}+\frac{1}{3^{2}}+\ldots+\frac{1}{3^{k}}\right)=\left(\frac{\mu+n+1}{3}\right)\left(\sum_{j=0}^{k-1} \frac{1}{3^{j}}\right)$. So the total support width is $(2)\left(\frac{\mu+n+1}{3}\right)\left(\sum_{j=0}^{k-1} \frac{1}{3^{j}}\right)=\mu+$ $n+1$.

\subsection{Comparison and Application}

Table 2 shows that the support size and continuity of proposed 3-point ternary scheme is same as 3-point ternary scheme introduced by [18]. It is also declared that the scheme introduced by [18] is $C^{2}$ for $\left(\frac{-1}{72}, \frac{7}{72}\right)$ while our 3-point scheme is $C^{2}$ for $\left(\frac{-1}{12}, \frac{11}{12}\right)$ which provides more freedom for curve designing. Support size of proposed 3-point ternary approximating scheme is smaller than 4-point ternary interpolating schemes [6] but gives the same order of derivative continuity. It is also mentioned that proposed 3-point scheme has larger interval of continuity with less computational cost than schemes [6] and [4]. 
In Table 2, we have pointed out that proposed 5-point ternary scheme is $C^{5}$ continuous while the 5-point ternary scheme of [15] is $C^{1}$.

Figure 2 shows the visual comparison of 3- and 5-point ternary interpolating schemes of Lian [15] with the proposed 3- and 5-point ternary approximating schemes. Figure $\mathbf{3}$ is exposed to show the role of shape parameter $\omega$ when proposed 3- and 5-point schemes applied on discrete data points. From this figure, we see that the behavior of the limiting curve acts as tightness when the choice of shape parameter vary from right to left in the interval $\left(\frac{-1}{12}, \frac{11}{12}\right)$.

\section{Conclusions}

The family of odd-point approximating schemes for curve design has established. Smoothness and approximation order of 3- and 5-point ternary schemes have been discussed. Support of family of odd-point ternary schemes has computed in general. It has been shown that proposed schemes are better then existing odd-point ternary schemes in the sense of smoothness. The family of even-point ternary approximating schemes will be studied in detail in the forthcoming paper.

\section{Acknowledgements}

The presented version of the paper owes much to the precise and kind remarks of the anonymous referee. This work is supported by the Indigenous Ph. D. Scholarship Scheme of Higher Education Commission (HEC) Pakistan

\section{References}

[1] C. Beccari, Gcasiola and L. Romani, “An Interpolating 4-Point $C^{2}$ Ternary Non-Stationary Subdivision Scheme with Tension Control," Computer Aided Geometric Design, Vol. 24, No. 4, 2007, pp. 210-219. doi:10.1016/j.cagd.2007.02.001

[2] M. F. Hassan, "Further Analysis of Ternary and 3-Point Univariate Subdivision Schemes,” Technical Report 599, University of Cambridge Computer Laboratory, ISSN 1476-2986, 2004.

[3] K. P. Ko, B.-G. Lee and G. Yoon, "A Ternary 4-Point Approximating Subdivision Scheme,” Applied Mathematics and Computation, Vol. 190, No. 2, 2007, pp. 15631573. doi:10.1016/j.amc.2007.02.032

[4] S. S. Siddiqi and K. Rehan, "Modified Form of Binary and Ternary 3-Point Subdivision Scheme," Applied Mathematics and Computation, Vol. 216, No. 3, 2010, pp. 970-982. doi:10.1016/j.amc.2010.01.115

[5] M. F. Hassan, N. A. Dodgson, "Ternary and Three-Point Univariate Subdivision Schemes,” In: A. Cohen, J. L.
Marrien, L. L. Schumaker, Eds., Curve and Surface Fitting: Sant-Malo 2002, Nashboro Press, Brentwood, 2003, pp. 199-208.

[6] M. F. Hassan, I. P. Ivrissimitzis, N. A. Dodgson and M. A. Sabin, "An Interpolating 4-Point $C^{2}$ Ternary Stationary Subdivision Scheme," Computer Aided Geometric Design, Vol. 19, No. 1, 2002, pp. 1-18. doi:10.1016/S0167-8396(01)00084-X

[7] F. Khan and G. Mustafa, "Ternary Six-Point Interpolating Subdivision Scheme," Lobachevskii Journal of Mathematics, Vol. 29, No. 3, 2008, pp. 153-163.

[8] D. Levin, “Using Laurent Polynomial Representation for the Analysis of Non-Uniform Binary Subdivision Schemes," Advances in Computational Mathematics, Vol. 11, No. 1, 1999, pp. 41-54. doi:10.1023/A:1018907522165

[9] G. Mustafa and F. Khan, "A New 4-Point $C^{3}$ Quaternary Approximating Subdivision Scheme," Abstract and Applied Analysis, Vol. 2009, 2009, Article ID 301967. doi:10.1155/2009/301967

[10] M. Sabin, "Eigenanalysis and Artifacts of Subdivision Curves and Surfaces, Tutorials on Multiresolution in Geometric Modeling,” In: A. Isle, E. Quak and M. S. Floater, Eds., Springer, Berlin, 2002, pp. 69-92.

[11] N. Dyn, K. Hormann, M. A. Sabin and Z. Shen, "Polynomial Reproduction by Symmetric Subdivision Schemes," Journal of Approximation Theory, Vol. 155, No. 1, 2008, pp. 28-42. doi:10.1016/j.jat.2008.04.008

[12] G. Mustafa, and A. R. Najma, "The Mask of $(2 b+4)$ Point $n$-ary Subdivision Scheme," Computing, Vol. 90, No. 1-2, 2010, pp. 1-14. doi:10.1007/s00607-010-0108-X

[13] J.-A. Lian, “On $a$-ary Subdivision for Curve Design: III. $2 m$-Point and $(2 m+1)$-Point Interpolatory Schemes," Applications and Applied Mathematics: An International Journal, Vol. 4, No. 2, 2009, pp. 434-444.

[14] K. Hormann and M. A. Sabin, "A Family of Subdivision Schemes with Cubic Precision," Computer Aided Geometric Design, Vol. 25, No. 1, 2008, pp. 41-52. doi:10.1016/j.cagd.2007.04.002

[15] A. Levin, "Polynomial Generation and Quasi-Interpolation in Stationary and Non-Uniform Subdivision Schemes," Computer Aided Geometric Design, Vol. 20, No. 1, 2003, pp. 41-60. doi:10.1016/S0167-8396(03)00006-2

[16] J.-A. Lian, "On $a$-ary Subdivision for Curve Design: II. 3-Point and 5-Point Interpolatory Schemes," Applications and Applied Mathematics: An International Journal, Vol. 3, No. 2, 2008, pp. 176-187.

[17] Y. Tang, K. P. Ko and B.-G. Lee, "A New Proof of Smoothness of 4-Point Deslauriers-Dubic Scheme," Journal of Applied Mathematics and Computing, Vol. 18, No. 1-2, 2005, pp. 553-562.

[18] J.-A. Lian, "On $a$-ary Subdivision for Curve Design: I. 4-Point and 6-Point Interpolatory Schemes," Applications and Applied Mathematics: An International Journal, Vol. 3, No. 1, 2008, pp. 18-29. 